\section{hommes}

\section{Hommes \& migrations}

Revue française de référence sur les dynamiques

migratoires

1315 | 2016

Ondes de choc

\title{
Le lien entre migration et terrorisme
}

Un tabou à déconstruire

Jean-Baptiste Meyer

\section{OpenEdition \\ 1 Journals}

\section{Édition électronique}

URL : http://journals.openedition.org/hommesmigrations/3715

DOI : 10.4000/hommesmigrations.3715

ISSN : 2262-3353

\section{Éditeur}

Musée national de l'histoire de l'immigration

\section{Édition imprimée}

Date de publication : 1 juillet 2016

Pagination : 49-57

ISBN : 978-2-919040-36-0

ISSN : $1142-852 X$

\section{Référence électronique}

Jean-Baptiste Meyer, « Le lien entre migration et terrorisme », Hommes \& migrations [En ligne], 1315 |

2016, mis en ligne le 01 juillet 2019, consulté le 21 décembre 2020. URL : http://

journals.openedition.org/hommesmigrations/3715; DOI : https://doi.org/10.4000/

hommesmigrations.3715 


\title{
LE LIEN ENTRE MIGRATION ET TERRORISME UN TABOU À DÉCONSTRUIRE
}

Par JEAN-BAPTISTE MEYER, directeur de recherche, laboratoire Population environnement développement (LPED), Aix-Marseille Université et Institut de recherche pour le développement (IRD).

\author{
Étudier directement le lien entre «terrorisme » et « migration » \\ invite à déconstruire les amalgames simplistes entre \\ religion, origine migratoire et radicalisation. Si les données \\ statistiques montrent que les terroristes qui ont frappé la \\ France sont en majorité français, elles font apparaître en creux \\ des problématiques existentielles bien connues des études \\ migratoires. Facteur parmi d'autres de la fabrique du radicalisme \\ musulman, nourri tout autant du mal-être social que du \\ sentiment de déracinement, l'empreinte du parcours migratoire \\ révèle l'asymétrie délétère d'une forme de mondialisation aux \\ conséquences inquiétantes.
}

Y a-t-il un lien entre migration et terrorisme, entre les flux migratoires contemporains et les attentats islamistes, entre les migrants et les djihadistes? La question semble tellement embarrassante qu'elle n'a pas encore été abordée de front. Immédiatement après les carnages, un sursaut d'évitement s'est même étendu comme une traînée de poudre : pas d'amalgames ! Ne pas confondre les musulmans avec les assassins, les immigrés avec les islamistes radicaux, les réfugiés avec des transfuges de Daesh : telle était la vision, largement partagée, tant le risque d'une réaction irrationnelle, sanglante en retour, hantait les esprits. Les semaines ont passé et il est temps maintenant de lever cette irrationa- lité - également risquée - qu'il y aurait à refuser d'étudier la question. Car il y a bien un lien entre la migration et ce terrorisme ; un lien empirique et visible... Le nier aujourd'hui reviendrait à renoncer à expliquer une part importante de ces événements. Et, ce faisant, à donner un surcroît de crédibilité à ceux qui, dans des amalgames tronqués mais concrets, forgent les fers de la discorde. Le présent texte est tout d'abord nourri d'informations et de réflexions issues des médias et des sciences sociales pour documenter l'analyse de ce lien entre migration et terrorisme islamiste. Mais il a également eu recourt à l'interview de personnes dont le profil éclaire l'engagement radical 
d'individus issus de l'immigration et en ouvre ainsi une explication sociologique. Il s'agit, en l'occurrence, d'individus issus de l'immigration en première ou deuxième génération, dont la trajectoire a été soigneusement relevée pour observer les bifurcations possibles ou avérées ${ }^{1}$. Enfin, tirant quelques conclusions des constats effectués, il suggère un positionnement différent pour surmonter les tensions, présentes et à venir, générées par ces événements répétés.

Quelques sondages ponctuels dans la sphère médiatique permettent de mesurer l'association entre les deux thèmes (migration et terrorisme) ainsi que ses représentations. Elle y est invoquée furtivement, de façon tardive mais croissante. Après les attentats de

Car il y a bien un lien entre la migration et ce terrorisme; un lien empirique et visible... Le nier aujourd'hui reviendrait

à renoncer à expliquer une part importante de ces événements. Et, ce faisant, à donner un surcroît de crédibilité à ceux qui, dans des amalgames tronqués mais concrets, forgent les fers de la discorde. novembre 2015, une recherche Internet par mots-clés, combinant des termes relatifs à la migration et au terrorisme, recueille de nombreuses références émanant souvent de la presse française. Une recherche identique effectuée quelques mois plus tôt - mais après les événements de janvier

- montrait que le sujet était alors le plus souvent éludé. En effet, la collecte d'informations rapportait essentiellement des références anglo-saxonnes, souvent produites au début des années 2000, après le 11 septembre 2001 : le lien mobilité-terrorisme était alors visiblement une préoccupation nord-américaine. Entre les deux massacres de 2015, entre le début et la fin de l'année, la crise migratoire avait explosé en Europe liant de façon inédite, au moins dans la communication, les deux termes et leurs problématiques. Elle produit cette floraison médiatique, murmure croissant mais parole non libérée, cependant, car lourde d'enjeux non élucidés.
Une telle évolution souligne l'importance cruciale du contexte historique et la coïncidence vécue et/ou perçue - des faits, des analyses et des interprétations.

\section{Migration et terrorisme, un lien dangereux}

Le lien entre migration et terrorisme est ouvertement devenu objet d'attention, même si la réflexion est restée limitée d'un point de vue conceptuel. Cette attention s'est en effet concentrée sur des constats empiriques, autour des interrogations suivantes : les personnes impliquées sont-elles migrantes ? Ont-elles infiltré les réseaux migratoires pour les utiliser? Proviennent-elles de milieux immigrés ? Mis à part les idéologues extrémistes qui ont sommairement fusionné les deux phénomènes, ces questionnements ont généré deux types de réaction : des constats embarrassés dans la presse à propos de possibles recoupements ou, de la part de certains académiques, des démonstrations de la négative - réponses certes humanistes, mais quelque peu naïves ou courtes. De fait, les terroristes islamistes en France et en Belgique ne sont généralement pas des migrants. C'est un fait très vite établi, une fois connue leur identité. Ils sont essentiellement de nationalité française ou belge. Le seul migrant intervenant dans les attentats de janvier 2015, par exemple, est au contraire un héros : c'est l'employé qui a sauvé des clients enfermés dans l'Hyper Cacher rappelle Philippe Fargues ${ }^{2}$. Plus tard, des individus venus de Syrie se sont glissés parmi les réfugiés - propres victimes de leurs violences en amont - transitant par la Grèce et l'Europe centrale, mais avec des passeports trafiqués. Par ailleurs, une étude statistique est mobilisée, avec toute la rigueur nécessaire. Elle souligne une corrélation entre les pays receveurs de migrants provenant de zones sensibles et les occurrences de terrorisme chez leurs hôtes. Mais la 


\section{/// Tableau 1 : Personnes impliquées dans des attentats terroristes en France de janvier 2012 à août 2016}

\begin{tabular}{|c|c|c|c|c|c|}
\hline NOM DES PERSONNES & $\begin{array}{l}\text { LIEUDE } \\
\text { NAISSANCE }\end{array}$ & MOBILITÉS & $\hat{A} G E$ & NATIONALITÉ & RÉSIDENCE \\
\hline BILAL HADFI & INCONNU & SYRIE & 20 & FRANÇAISE & BRUXELLES \\
\hline SID AHMED GHLAM & ALGÉRIE & TURQUIE & 24 & ALGÉRIENNE & PARIS \\
\hline AYOUB EL-KHAZANI & MAROC & EUROPE & 26 & MAROCAINE & BELGIQUE \\
\hline MOHAMED MERAH & FRANCE & AFGH., PAK. & 24 & $\begin{array}{l}\text { FRANCO- } \\
\text { ALGÉRIENNE }\end{array}$ & TOULOUSE \\
\hline ISMAEL OMAR MOSTEFAI & FRANCE & SYRIE & 29 & FRANÇAISE & CHARTRES \\
\hline SAMY AMIMOUR & FRANCE & SYRIE & 28 & FRANÇAISE & DRANCY \\
\hline SAID KOUACHI & FRANCE & YÉMEN & 34 & FRANÇAISE & REIMS \\
\hline CHÉRIF KOUACHI & FRANCE & YÉMEN? & 32 & FRANÇAISE & GENNEVILIERS \\
\hline AMEDY COULIBALY & FRANCE & INCONNUES & 32 & FRANÇAISE & BAGNEUX \\
\hline YASSIN SALHI & FRANCE & SYRIE? & 35 & FRANÇAISE & BESANÇON \\
\hline MUSTAPHAMOKEDDEM & FRANCE & SYRIE & 21 & FRANÇAISE & TOULOUSE \\
\hline HAKIM MARNISSI & FRANCE & INCONNUES & 25 & FRANÇAISE & TOULOUSE \\
\hline LAROSSI ABBALLA & FRANCE & INCONNUES & 25 & INCONNUE & INCONNUE \\
\hline SALAHABDESLAM & BELGIQUE & SYRIE? & 26 & FRANÇAISE & MOLENBEEK \\
\hline BRAHIM ABDESLAM & BELGIQUE & SYRIE? TURQ. & 31 & FRANÇAISE & MOLENBEEK \\
\hline ABDELHAMID ABAAOUD & BELGIQUE & SYRIE & 28 & $\begin{array}{c}\text { BELGO- } \\
\text { MAROCAINE }\end{array}$ & MOLENBEEK \\
\hline $\begin{array}{l}\text { MOHAMED LAHOUAIEJ } \\
\text { BOULEL }\end{array}$ & TUNISIE & INCONNUES & 31 & TUNISIENNE & NICE \\
\hline ADEL KERMICHE & FRANCE & $\begin{array}{l}\text { TENTATIVE } \\
\text { SYRIE }\end{array}$ & 19 & $\begin{array}{l}\text { FRANCO- } \\
\text { ALGÉRIENNE }\end{array}$ & $\begin{array}{l}\text { SAINT-ÉTIENNE- } \\
\text { DU-ROUVRAY }\end{array}$ \\
\hline ABDEL MALIK PETITJEAN & FRANCE & TURQUIE & 19 & FRANÇAISE & AIX-LES-BAINS \\
\hline
\end{tabular}


migration s'avère aussi, de la même façon, être un facteur de réduction proportionnelle de telles violences, par sa propagation d'effets bénéfiques, naturellement pacificateurs ${ }^{3}$. Dans une vision moins irénique, l'approche statistique est aussi mobilisée pour souligner combien les chiffres interdisent des assimilations rapides. Sur près de 5 millions de Français de religion musulmane, à peine un millième, soit environ 5 000, est répertorié comme djihadiste potentiel ou avéré. Et encore, parmi ces derniers, y en a-t-il un certain nombre, probablement plus du quart, qui sont français " de souche $e^{4}$ ».

Les différentes démonstrations rapportées invoquent les chiffres pour prouver qu'un lien entre les migrations et les actes terroristes perpétrés est insignifiant. Pourtant, l'argument statistique peut se retourner. Car, au-delà d'une représentativité par la loi des grands nombres, il existe des possibilités de mesurer la probabilité que des variables soient associées en produisant des conditions expérimentales contrôlées. En biologie médicale, par exemple, c'est toute la différence entre les études épidémiologiques sur de

L'histoire comparable

d'enfants de migrants

aux trajectoires d'abord

ressemblantes mais

aux débouchés opposés nous montre comment

l'incompatible est

modulable et les passifs

reconvertibles. vastes populations et les essais cliniques sur des petits groupes. En sciences sociales, les observations du premier type sont courantes mais les expérimentations du second sont exceptionnelles.

Or les attentats ont produit ces conditions exceptionnelles. Ils ont isolé des acteurs et rendu possible une analyse particulière. Ils montrent sans équivoque que, s'il y a peu de migrants parmi les terroristes, ils sont tous en revanche issus de l'immigration. Leur origine géographique ou celle de leurs parents se situe au Maghreb, dans des pays dont ils peuvent avoir gardé la nationalité. Ils revendiquent tous l'islam, d'une façon ou d'une autre. Leur mobilité spatiale est notoire avec des séjours en Europe mais aussi, bien souvent, en Syrie, en Afghanistan, au Pakistan, en Turquie et au Yémen ${ }^{5}$. Les recoupements systématiques de ces quelques variables entre tous ces profils ne sont guère le fruit du hasard. Ils marquent bien des caractéristiques partagées dont il convient de saisir l'incidence sur leurs actes.

\section{Malaise social et revendications religieuses}

En partant de ce constat initial de la migration et de la mobilité caractéristiques de la trajectoire personnelle des terroristes, les explications diffèrent. Certaines, d'inspiration économique, relèvent l'importance de la demande, avec ses instances (Daesh, AQMI, etc.) recrutant les personnes idoines, puis façonnant leurs profils. Elle insiste sur les véritables « centrales » de production de ces individus mortifères ${ }^{6}$. Relevant qu'il s'agit de personnes fréquemment assez qualifiées, le constat est fait que leurs « compétences » djihadistes sont élaborées et développées dans des lieux particuliers où ils transitent, jeunes et en cours de formation.

Une étude sociologique approfondie menée à la fin des années 2000 révèle une prévalence du profil d'ingénieur chez les islamistes radicaux? Elle confirme que la majorité des terroristes islamistes ayant opéré dans les pays occidentaux sont résidents et même souvent citoyens de ces derniers. Mais elle relève, sur un échantillon de 13 de ces pays sélectionnés pour leur disponibilité de données, que la qualification socioprofessionnelle est généralement plus élevée lorsque les individus viennent de l'étranger que lorsqu'ils sont issus de la population locale. Il y aurait donc, selon ces auteurs, une "lumpen " catégorie parmi les contingents terroristes. Cette distinction est effectuée également par les chercheurs s'attachant 
à mieux comprendre ce qui se passe sur le versant de l'offre de cette main-d'œuvre terroriste. Parmi les personnes radicalisées, deux profils émergent : ceux provenant typiquement de la banlieue et les autres des classes moyennes, souvent urbaines, provinciales ou non ${ }^{8}$; les premiers sont plus généralement issus de l'immigration que les seconds, d'ascendance française croissante ${ }^{9}$. Cette radicalisation apparaît comme la résultante d'un intense malêtre social, d'une citoyenneté introuvable dans le contexte actuel mondialisé ${ }^{10}$ et lié au déracinement de cette population dans la modernité ${ }^{11}$. Cet extrême désarroi serait concentré essentiellement dans la deuxième génération de l'immigration et révélerait un problème d'intégration : porteuse d'un héritage musulman mal transmis, elle répondrait par la production d'un « islam de rupture ${ }^{12}$ ». On retrouve là un thème proche d'un classique de la sociologie de l'immigration : celui de la double absence, d'un vide interculturel, conséquence d'un entre-deux, mal vécu, mal assumé, creuset de souffrance et d'exclusion ${ }^{13}$.

Cependant, dans cette vision, c'est le malaise social et générationnel qui prime et qui surdétermine le choix de la radicalisation. Celle-ci se porte sur l'islam comme forme à investir et Olivier Roy parle ainsi d'islamisation de la radicalité pour bien souligner que l'aspect religieux est second par rapport à une revendication plus globale qui s'exprime à travers lui. Cette revendication fondamentale ne serait guère nouvelle et imprégnait aussi les mouvements terroristes de la deuxième moitié du XXe siècle, qui s'incarnaient dans une idéologie subversive. Les Brigades rouges italiennes, la Fraction armée rouge en Allemagne de l'Ouest, Action directe en France avaient bien des caractères similaires, notamment dans la jeunesse de ses membres et le rejet d'un ordre mondial/occidental dominant. Mais il y a une différence fondamentale : elles étaient justement d'un pays alors que les

mouvements djihadistes d'aujourd'hui sont transnationaux. Leurs territoires sont globaux et leurs réseaux sont planétaires. Ce trait n'a pas échappé à Jean Baudrillard qui voit, dans les attentats du 11 septembre 2001, l'effet de la mondialisation dans les développements du terrorisme $e^{14}$. Si les cibles ponctuelles des djihadistes peuvent être un État particulier pour des raisons de conjoncture, l'objet de leur lutte c'est la société mondialisée dans ses formes perçues comme antagoniques avec l'islam.

Marcel Gauchet, entre autres, a tenté de penser le contenu de cette religion pour y déceler les explications des revendications islamistes radi-

On voit dans le profil initial de cette personne de nombreux traits renvoyant à celles radicalisées dans des conditions identiques : origine immigrée modeste, précarité financière et sociale, difficultés familiales et personnelles mais également la débrouille dont ils font preuve. cales $^{15}$. Des explications qui dépasseraient le cadre externaliste exposé précédemment, où le primat du social réduit le religieux à un investissement de forme. Ainsi, dernière religion révélée, version ultime du monothéisme, l'islam marque par son histoire un point d'aboutissement. Mais il est porté par une population souvent opprimée depuis plusieurs siècles dans son rapport avec les autres. Il se lit dans ses textes fondateurs, des messages qui apportent une contradiction à la mondialisation contemporaine dans ses aspects matérialistes et consuméristes. Mais ses hérauts ne sont pas en position de faire valoir et respecter ces points de vue. Cette impuissance ravive le sentiment de subordination injustifiée, enracinée dans les cultures du monde arabe ${ }^{16}$.

Il y a dans cette dernière approche une tentative d'expliquer pourquoi l'islam "fixe » aujourd'hui une revendication qui peut dégénérer à l'extrême. Ses composantes symboliques comme son histoire sont mobilisées pour fournir les facteurs 
explicatifs, internes à cette religion et constitutifs de la causalité des actes commis. Tout en se gardant d'essentialiser des propriétés de l'islam, il convient de retenir cette association historique entre ce qu'il représente, l'émergence d'une société mondiale et le conflit qui s'exprime à C'est un premier enseignement

si l'islam incarne aujourd'hui souvent une réaction à la forme dominante de la mondialisation actuelle, c'est en raison d'une histoire marquée par la violence et l'imposition unilatérale d'une telle forme exogène dans son espace originaire. travers l'ultra-violence des attentats. Mais cette association n'est pas univoque. L'histoire comparable d'enfants de migrants aux trajectoires d'abord ressemblantes mais aux débouchés opposés nous montre comment l'incompatible est modulable et les passifs reconvertibles. Cet exercice nous éloigne d'un déterminisme psychologique où l'acte éminemment (anti)social du terrorisme serait fondu dans une version individuelle où l'altérité ne serait plus que le jouet de la subjectivité. La presse s'est en particulier largement faite écho d'une telle vision dans ses descriptions et commentaires sur les profils border line de Merah ou Lahouaiej Boulel.

\section{Double absence et double présence}

Une récente recherche menée par Aziz Nafa sur les entrepreneurs issus de la diaspora algérienne (principalement localisés en France, mais pas seulement) et qui développent des activités avec leur pays d'origine révèle des cas édifiants sur la tension sociale, dynamique et aléatoire, que génère leur parcours familial migratoire ${ }^{17}$. On y voit comment des individus - issus de la deuxième génération notamment - accommodent leur passé familial, leur histoire personnelle, leurs projets socioprofessionnels, leurs engagements religieux et autres.
Clairement, dans leurs récits, la place même de l'économie et de la religion se négocie et s'articule, sans conflits inhérents. On sort ainsi d'une opposition fondamentale, souvent invoquée, entre des systèmes de valeurs perçus comme incompatibles. Le capitalisme et l'islam contemporains apparaissent alors tout à fait miscibles dans des conditions locales travaillées par les acteurs au cas par cas, où l'un et l'autre se recomposent ${ }^{18}$. Dans des versions toujours contextualisées, ils traduisent leurs combinaisons respectives en synergies plutôt qu'en contradictions.

Cette enquête sur les profils de plusieurs dizaines de ces entrepreneurs de la diaspora algérienne en France peut-être condensée dans le cas représentatif de Farid ${ }^{19}$. Sa trajectoire éclaire profondément comment il est devenu ce qu'il est aujourd'hui mais aussi ce qu'il aurait pu devenir... Elle peut être mise en perspective avec celles - rapportées par la presse - des vies difficiles des terroristes identifiés au tableau 1.

«M. Farid: Moi je suis issu d'une famille d'immigrés algériens. Mes parents sont arrivés en France très jeunes. En fait ils sont arrivés pendant la guerre d'Algérie. Ma mère était une fille orpheline. Mon père était ouvrier textile et ma mère travaillait très tard. Donc j'ai fait mes études : l'école maternelle classique, l'école primaire sans soucis, le collège, je ne peux pas dire sans soucis parce que la sixième était un peu dure. À l'époque, mon père venait d'acheter une boucherie. À l'usine où il travaillait, la crise textile était arrivée, donc il avait essayé d'ouvrir une boucherie. Ça n'a pas marché. C'était une catastrophe économique familiale, mes parents ont failli divorcer. Quand on est en sixième, on n'est pas forcément bien. Le chômage, le surendettement de la maison, etc. Dès l'âge de 15 ans, j’ai décidé d'être autonome financièrement. J'ai commencé à travailler, j'ai trouvé des boulots à droite et à gauche... J'avais toujours un petit business quelque part qui me permettait de payer mes vêtements, mon école etc., et

17. Aziz Nafa, "Rôle de la diaspora algérienne dans le développement de l'entrepreneuriat et le transfert de connaissances ", thèse de doctorat Aix-Marseille Université, 2015. 18. Mohamed Madoui, reprenant le fameux travail de Max Weber sur "l'éthique protestante et l'esprit du capitalisme », montre que l'islam des entrepreneurs maghrébins n'est en rien moins efficace pour accompagner leur activité commerciale ou industrieuse. Voir Mohamed Madoui, Entreprises et entrepreneurs en Algérie et dans l'immigration. Essai de sociologie économique, Paris, Karthala, 2012. 19. Interview réalisée en 2013 dans le cadre de la thèse d'Aziz Nafa. 
d'aider un peu mes parents. Ensuite, je suis rentré au lycée. En seconde, cétait un peu difficile. J'ai fait un bac spécialisé en math et en physique, à l'époque qu'on l'appelait “F3", et l'école technique. J'avais peur d'aller en $S$ parce que je n'aimais pas beaucoup travailler. »

On voit dans le profil initial de cette personne de nombreux traits renvoyant à celles radicalisées dans des conditions identiques : origine immigrée modeste, précarité financière et sociale, difficultés familiales et personnelles mais également la débrouille dont ils font preuve ${ }^{20}$. La séquence suivante, consacrée à l'insertion professionnelle, est également éloquente.

"M. Farid: Après mon bac F3, je me suis inscrit à la fac en cours d'informatique à l'USTN de Lille. Cela me plaisait moyennement et, à l'époque, j'avais une très grande activité associative. Enfin, j'avais une certaine activité politique et jétais au mouvement des jeunes socialistes de ma ville. J'ai fait durant l'été une mâttrise en gestion sociale en deux ans. À l'issue de ces deux ans, j'ai vu que le social ne me convenait pas. En fait, le décès de mon père avant la fin de mon master a suscité beaucoup de remise en question. Il y avait de nouveau des problèmes financiers à la maison et une ambiance de mort. Mon père était jeune, il n'avait que 45 ans. Je suis allé voir à Paris si je pouvais trouver du travail... Rapidement, j'ai signé mon contrat en informatique. En 1998, c'était le plein boom de l'informatique. J'étais surpris d'avoir 1100 à 1200 euros de salaire. Pour moi, c'était énorme. »

Dans un parcours qui n'a rien d'élitiste, Farid avance, en butte aux accidents de la vie, mais aussi aux chances offertes, qui sont autant de bifurcations possibles. Il se déplace en France et en Europe du Nord pour s'offrir des opportunités. Ses premiers pas sont durs et ses gratifications modestes comme beaucoup de jeunes de sa génération. Pourtant, elles lui offrent la possibilité de débuter, de se faire connaître et reconnaitre. Il entre à Wanadoo puis décide de fonder sa propre entreprise de développement de sites Internet. C'est dans le prolongement de celle-ci qu'il choisit de s'installer quelques années plus tard en Algérie.

«M. Farid: Un changement a eu lieu depuis la dernière fois où je suis allé en Algérie, en 2004. J'étais au village et on m'a appelé "lémigré". Cela m'a un peu choqué en fait. C'était tous des cousins... Je les ai laissés et j'ai pris du recul. Quand vous êtes en Algérie, vous êtes un émigré, vous nêtes pas Algérien. Quand vous êtes en France, vous êtes un immigré, vous n'êtes pas français. C'est pour cela que certains jeunes qui sont un peu plus fragiles psychologiquement pensent: "Non ce n'est pas ça, je suis musulman, donc je vais être un islamiste." Quelque part, logiquement, il n'y a que l'islam qui me donne une identité. Et moi, je me suis dit : je suis les deux. Et je le prends

vraiment comme une force, un avantage énorme, parce qu'aujourd'hui, ma culture algérienne est limitée aux pratiques qu'on a à la maison. Avec mes grandsparents, ou mes parents, la façon de penser est typiquement algérienne. Quand je viens en Algérie, je comprends d'où ils viennent. La culture française est plus en rapport avec lorganisation du travail, la façon de voir les choses, etc. Et cela, en définitive, je ne sais pas si c'est français ou si c'est Farid. Je pense que c'est plus Farid que français parce que c'est un pays extrêmement pessimiste. "

Apparaissent ici très clairement cette sensation d'une double discrimination/exclusion et la question identitaire qu'elle génère. L'analogie avec l'islamisme radical est faite spontanément par Farid, interviewé en 2013, par conséquent avant les événements terroristes. Il relève cependant également l'aubaine que représente son parcours interculturel, avec des valeurs et des compétences acquises en France, transférables et valorisables ailleurs. Il donne aussi la pleine mesure de la position individuelle par rapport aux contextes/cultures de l'Algérie et de la France.

À l'instar du parcours de Farid, les autres entrepreneurs interviewés - hommes ou femmes - s'ins- 
crivent dans cette asymétrie entre les deux univers mis en contact à travers leur personne biculturelle. Ils/elles resituent leur histoire dans celle, collective, de deux pôles migratoires très dépendants du rapport postcolonial. Cette asymétrie, historiquement produite, est directement constitutive de la tension vitale et sociale des individus concernés, de leurs difficultés particulières et des possibilités de dérapage existentiel qu'elle engendre. Mais cette différence de potentiel, entre Nord et Sud, constitue aussi, de la même façon, l'opportunité inédite de fertilisation croisée que ces amphibiens culturels peuvent réaliser. Nul déterminisme univoque mais plutôt une disjonction relative aux parcours des acteurs, aux alternatives qui s'offrent à eux et aux choix qu'ils effectuent.

\section{Des relations sociales mondiales asymétriques}

Peut-on regrouper quelques enseignements de tous les éléments présentés dans les pages qui précèdent ? Tout d'abord, il faut prendre au sérieux la référence que les propres acteurs font à l'islam. Que les terroristes soient ou non de mauvais ou de récents pratiquants importe peu : on ne peut réduire leur revendication religieuse à un prétexte qui masquerait simplement d'autres motifs. Les parcours retracés montrent que l'islam est significatif dans la vie des personnes, ne serait-ce que par leur histoire. Que les acteurs (entrepreneurs ou djihadistes) s'en réclament, pour leurs activités productives ou destructives, selon la vocation qu'ils se donnent, est un signe éloquent d'autodétermination, d'identification. Que l'islam traduise alternativement ou simultanément une "globalisation du ressentiment ${ }^{21} »$ ou au contraire une vitalité identitaire dans un espace transméditerranéen fertile, il apparaît comme un catalyseur d'énergie sociale $^{22}$. S'il incarne souvent le ressentiment plu- tôt que l'espoir vis-à-vis de l'Occident, c'est aussi parce que leurs rapports sont marqués, dans l'histoire moderne et dans le présent, par une relation asymétrique. Cette dernière a étouffé les possibilités de développement dans le monde arabo-musulman, éventuellement différentes de celles issues de l'Occident ${ }^{23}$. C'est un premier enseignement : si l'islam incarne aujourd'hui souvent une réaction à la forme dominante de la mondialisation actuelle, c'est en raison d'une histoire marquée par la violence et l'imposition unilatérale d'une telle forme exogène dans son espace originaire.

Cette asymétrie, inscrite dans la géographie de la Méditerranée, est également le moteur des migrations et des mobilités passées et actuelles. Les hommes se déplacent vers certains centres d'activité, organisés par des groupes à leur profit. L'interdépendance est alors inégale, servant, plus ou mieux, certaines parties que d'autres. Cette relation de subordination, d'une partie à une autre, est éminemment politique. Elle peut donc être traitée, par le dialogue, mais aussi par la force. La colonisation a usé des deux modalités et a maintenu l'asymétrie. Elle s'est élargie à d'autres entités dans le monde postcolonial d'aujourd'hui. Ainsi, les ÉtatsUnis et l'Allemagne sont l'objet de la vindicte de terroristes issus de pays avec lesquels ils entretiennent une relation migratoire intense (Afghanistan, Syrie, Turquie, etc.), tout comme la France avec ses anciennes colonies maghrébines. Mais, comme le montre cette extension contemporaine, c'est moins la relation historique séculaire qui est en jeu qu'une problématique (géo)politique tout à fait actuelle ${ }^{24}$. C'est un deuxième enseignement : si l'asymétrie procède d'une relation historique particulière, celle de la colonisation, elle la transcende aujourd'hui et mérite d'être abordée sous ses formes actuelles offrant aussi des modalités inédites de traitement.

Enfin, un troisième enseignement porte sur la question sociale : si la ghettoïsation des banlieues, 
l'exclusion et la précarité affectant les milieux marqués par l'immigration constituent une part de l'explication des violences terroristes, elle ne s'y réduit pas. Le recrutement djihadiste va bien audelà avec des profils qualifiés, mobiles, professionnels, entre autres. Et bien des enfants d'immigrés construisent au contraire positivement une mondialisation intégrée. Au-delà des options militaires/sécuritaires aux résultats limités, celles émanant du social ne peuvent espérer tout résoudre. Il faut ainsi penser à d'autres voies également, peutêtre moins restreintes à des dispositifs étatiques traditionnels...

\section{Conclusion}

En définitive, la migration apparaît bien comme un élément fondamental de la situation actuelle. Elle permet de mettre en perspective les événements et d'expliquer un peu mieux un présent sidérant. Elle reflète une asymétrie planétaire - au-delà d'un problème d'insertion sociale locale - que nos États ne parviennent pas à résoudre.

Alors, une question grave reste en suspens : sommes-nous face à une fatalité qui nous échappe? Le terrorisme du XXIe siècle est-il la transposition au niveau mondial - par la migration massive survenue à la fin de l'époque précédente - d'une incontournable dialectique dominant-dominé, comme le suggère Baudrillard ? Y a-t-il moyen de dépasser cette situation issue de la relation postcoloniale qui marque profondément la mondialisation actuelle? Certaines options se dessinent dans les réflexions actuelles autour du cosmopolitisme. Il s'agirait de sortir d'une vision que l'on peut qualifier d'impériale, où s'impose une version universelle de la vie sociale. Car, sur ses marges, l'empire laisse toujours quelques entités insoumises et récalcitrantes, susceptibles de velléités de contestation. La contention de celles-ci est coûteuse, inique et paralysante ${ }^{25}$.
Elle reste aussi toujours précaire et limitée tant on ne peut les éliminer complètement.

Pour dépasser ce paradigme actuel de la mondialisation, il faut penser à une cohabitation inédite de plusieurs modes géopolitiques, pas nécessairement commensurables entre eux. Nous ne serions plus dans une logique exclusive, de l'un ou l'autre, mais inclusive, de l'un et l'autre ${ }^{26}$. Nous ne concevrions plus les solutions dans le cadre de l'universalisme mais d'un pluri-versalisme ${ }^{27}$. Selon cette révision fondamentale de la philosophie politique ainsi mise en question, c'est le sens de la révélation, consignée dans les religions du livre, mais aussi celui du discours des Lumières, dont les prétentions universelles méritent d'être reconsidérées et retraduites. Est-ce utopie que de vouloir imaginer une telle tolérance? Beaucoup le pensent, en réaffirmant des valeurs propres et normatives... Mais, à l'affirmative, devra-ton alors souffrir indéfiniment la violence secrétée aux nouvelles marges - extérieures et intérieures - d'un empire mondial ? 